\title{
1/f Noise modeling of InAs/GaSb superlattice mid- wavelength infrared detectors
}

\author{
${\text { Lukasz } \text { Ciura }^{1} \text { (D) Andrzej Kolek }}^{1} \cdot$ Jarosław Jureńczyk $^{2} \cdot$ \\ $\mathrm{Krzysztof} \mathrm{Czuba}^{2} \cdot$ Agata Jasik $^{2}$ - Iwona Sankowska ${ }^{2}$. \\ Janusz Kaniewski ${ }^{2}$
}

Received: 29 August 2017/ Accepted: 23 December 2017/Published online: 30 December 2017

(C) The Author(s) 2017. This article is an open access publication

\begin{abstract}
The empirical 1/f noise model for $\mathrm{p}^{+}$-p-n infrared detector made of type-II InAs/ $\mathrm{GaSb}$ superlattice material is presented. It is shown that $1 / \mathrm{f}$ noise magnitude can be accurately estimated if dark current contributions are determined and noise coefficients are known. It is found that the shunt, the bulk generation-recombination, and the trap-assisted tunneling currents contribute to the total $1 / \mathrm{f}$ noise. No $1 / \mathrm{f}$ noise connected with the diffusion and the band-to-band tunneling currents is observed.
\end{abstract}

Keywords InAs/GaSb superlattice $\cdot$ Dark current modeling $\cdot 1 / \mathrm{f}$ Noise modeling

\section{Introduction}

The noise of a photovoltaic detector defines detectivity one of its figures of merit. The detectivity is expressed with the formula $D^{*}=R_{I} A^{1 / 2} / i_{n}$, which includes current responsivity $R_{I}$, detector area $A$, and noise current $i_{n}$. Therefore, the calculation of detectivity requires noise measurements or at least noise estimation. Usually, in such noise estimation, only the thermal and the shot noise are taken into account if the detector is biased

This article is part of the Topical Collection on Numerical Simulation of Optoelectronic Devices, NUSOD' 17.

Guest Edited by Matthias Auf der Maur, Weida Hu, Slawomir Sujecki, Yuh-Renn Wu, Niels Gregersen, Paolo Bardella.

Łukasz Ciura

lciura@prz.edu.pl

1 Department of Electronics Fundamentals, Rzeszow University of Technology, Al. Powstańców Warszawy 12, 35-959 Rzeszow, Poland

2 Institute of Electron Technology, Al. Lotników 32/46, 02-668 Warsaw, Poland 
(Callewaert et al. 2014; Khoshakhlagh et al. 2010; Martyniuk et al. 2012, 2016; Rodriguez et al. 2007). The noise consideration of a biased detector should also include the $1 / \mathrm{f}$ noise, which can significantly reduce detectivity in the low-frequency regime. Still, the general theory of 1/f noise is lacking; however, several models of 1/f noise addressed for infrared devices exist (van der Ziel 1986; Kleinpenning 1983; Kinch et al. 2009). Most of them concern $\mathrm{HgCdTe}$-based devices and are related to the specific detector designs. Therefore, the precise evaluation of the detectivity still requires $1 / \mathrm{f}$ noise measurements, which are expensive and time-consuming. Any attempt, which aims at the prediction of $1 / \mathrm{f}$ noise level, is then valuable because it can save experimental effort and makes the estimation of the detectivity more reliable. It is assumed that in a quite universal way the total $1 / \mathrm{f}$ noise p.s.d. can be calculated as the sum of the contributions introduced by each dark current component of the detector (Bae et al. 2000; Bajaj 1992). We have recently shown (Ciura et al. 2016) that: (i) the diffusion current does not make observable contribution to $1 / \mathrm{f}$ noise; (ii) the shunt current $I_{s h}$, the bulk generation-recombination (g-r) current $I_{g-r}$, and the tunneling current $I_{\text {tun }}$ (trap-assisted or band-to-band) make significant contributions to 1/f noise. Finally, the total power spectral density (p.s.d.) of 1/f noise can be described by:

$$
S_{i}(f)=\left(\alpha_{s h} I_{s h}^{2}+\alpha_{g-r} I_{g-r}^{2}+\alpha_{t u n} I_{t u n}\right) / f,
$$

where $\alpha$ is the $1 / \mathrm{f}$ noise coefficient associated with each current component. Making use of Eq. (1) requires resolving dark current into its components, which can be done employing current modeling. Then the extraction of the $1 / \mathrm{f}$ noise coefficient should be done based on $1 / \mathrm{f}$ noise measurements. In this paper, we introduce the $1 / \mathrm{f}$ noise model, decompose the last term of Eq. (1) into trap-assisted or band-to-band 1/f noise constituents, and use developed model to estimate $1 / \mathrm{f}$ noise in a InAs/GaSb superlattice (SL) detector in the wide range of voltage bias and temperature.

\section{Detector}

The 1/f noise model is formulated and adjusted for the real device \#E505, presented in Fig. 1. Detector was grown by Molecular Beam Epitaxy at the Institute of Electron Technology, Warsaw, Poland. Basically, the detector has $\mathrm{p}^{+}-\mathrm{p}-\mathrm{n}$ architecture, where $\mathrm{p}$ is the absorber, $\mathrm{p}^{+}$and $\mathrm{n}$ are the electron and the hole contacts, respectively. These three layers are made of InAs/GaSb SL with basic period of 10 monolayers/10 monolayers (ML). Detector was grown on $2^{\prime \prime}$ GaSb substrate with three GaSb buffer layers, doped with beryllium. The p contact consists of 40 periods of $10 \mathrm{ML} / 10 \mathrm{ML}$ InAs/GaSb SL, doped with beryllium to $N_{A}=5 \times 10^{17} \mathrm{~cm}^{-3}$ (doping in GaSb layers). The absorber consists of 400 periods of $10 \mathrm{ML} / 10 \mathrm{ML} \mathrm{SL}$, doped with tellurium to $N_{D}=5 \times 10^{17} \mathrm{~cm}^{-3}$ in GaSb layers. The $\mathrm{n}$ contact consists of 60 periods of $10 \mathrm{ML} / 10 \mathrm{ML}$ SL, which is tellurium doped to $N_{D}=5 \times 10^{17} \mathrm{~cm}^{-3}$ in InAs layers. The contact is followed by the tellurium-doped InAs cap layer. The noise and dark current measurements, which are presented in Figs. 2, $3,4,5,6,7$ and 8, are conducted with a transimpedance amplifier which was described elsewhere (Ciura et al. 2014). The example noise p.s.d. functions, measured for different reverse bias voltages at constant temperature $T=77 \mathrm{~K}$, are shown in Fig. 2. Generally, they have $1 / f$ noise shape. Deviations appear for the low bias and frequency higher than a few $\mathrm{Hz}$ due to the contributions from the $\mathrm{g}-\mathrm{r}$ noise which have Lorentzian-like p.s.d. $S_{i} \sim A /\left(1+\left(f / f_{c}\right)^{2}\right)$, where $A$ and $f_{c}$ are the constants. At $1 \mathrm{~Hz}$, the p.s.d.s are not affected 


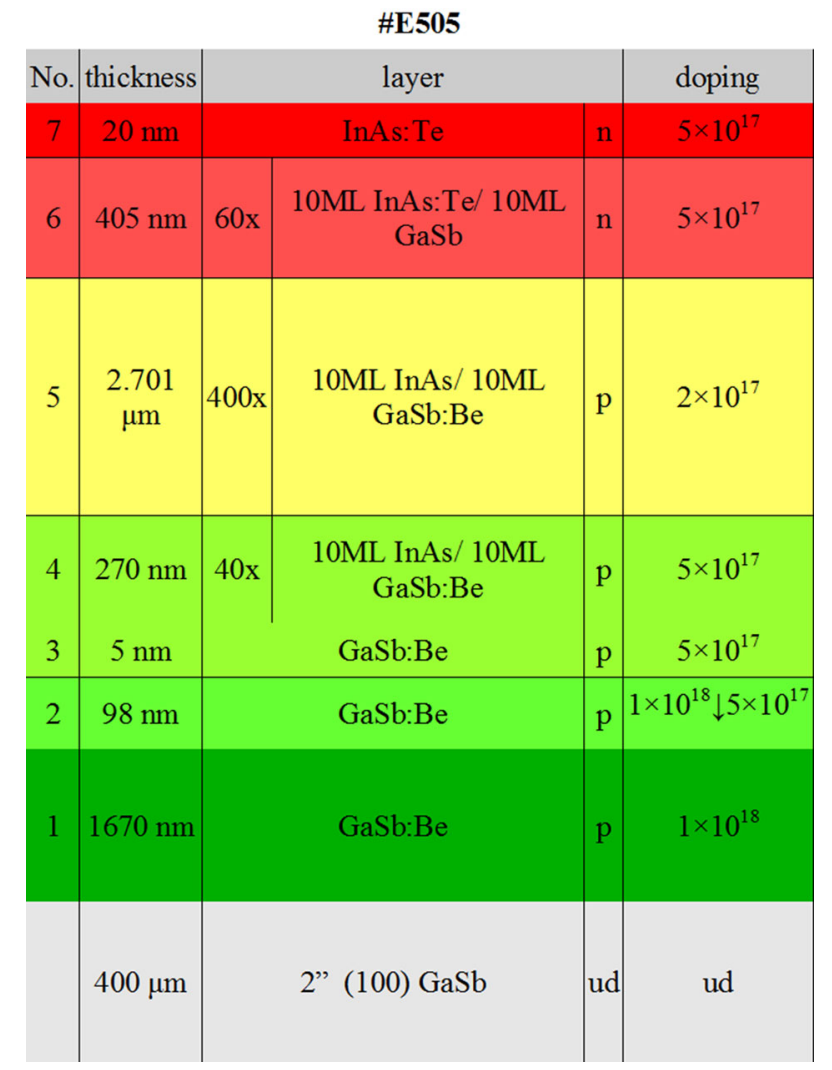

Fig. 1 Layers sequence, doping, and width in the device \#E505

Fig. 2 Power spectral densities versus frequency for different reverse voltage bias at constant temperature $T=77 \mathrm{~K}$

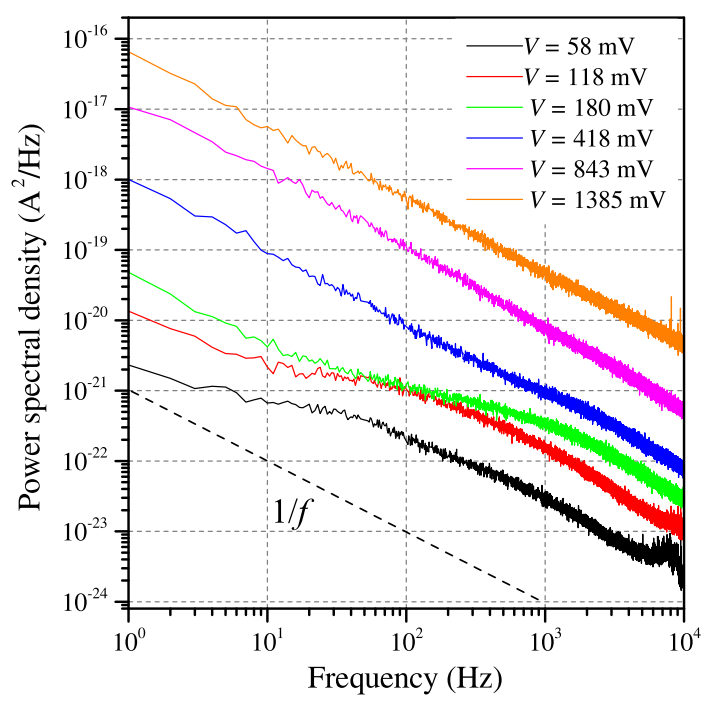


Fig. 3 Plot of squared ratio of area and detector capacitance ratio versus bias voltage. The reduced carrier concentration is determined as the fitting parameter
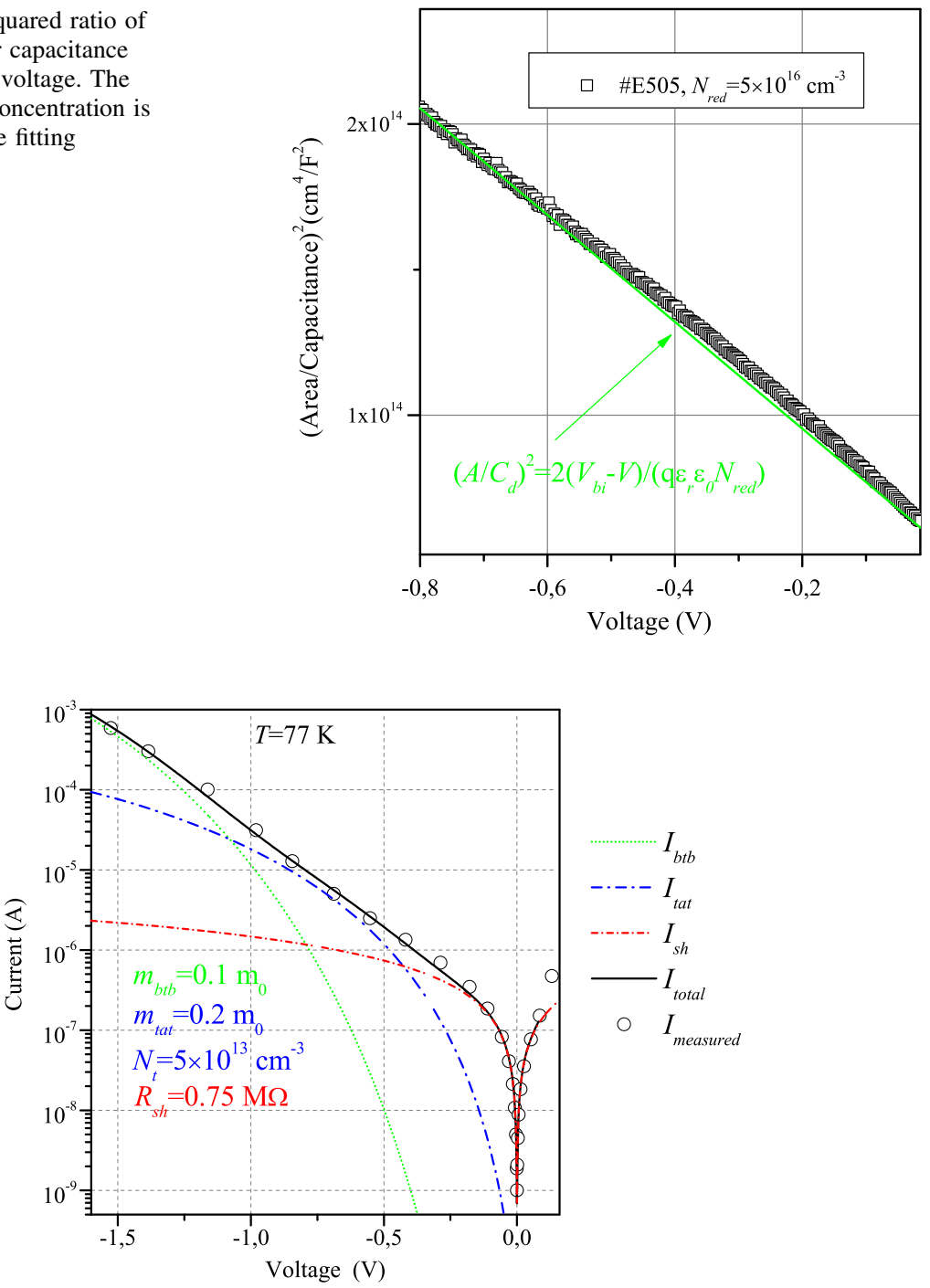

Fig. 4 Dark current components of the detector versus bias voltage at $T=77 \mathrm{~K}$

by such g-r noise so that the value $S_{i}(1 \mathrm{~Hz})$ is assumed as $1 / f$ noise intensity measure and used in all further analyzes.

\section{Dark current modeling-the method}

There are several works which deal with dark current modeling of SL-based devices (Ciura et al. 2016; Czuba et al. 2017; Gopal et al. 2008; Martyniuk et al. 2012; Nguyen et al. 2004; Peng et al. 2015). However, there is no electronic transport model, dedicated for superlattice-based devices so far. For this reason, commonly used models of dark currents 


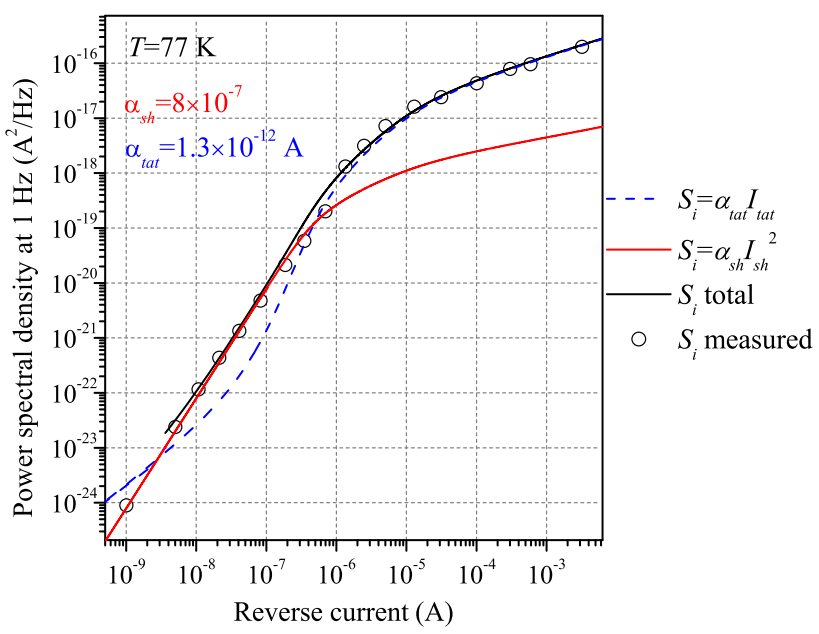

Fig. 5 Measured 1/f noise and its modeled contributions versus total current at $T=77 \mathrm{~K}$

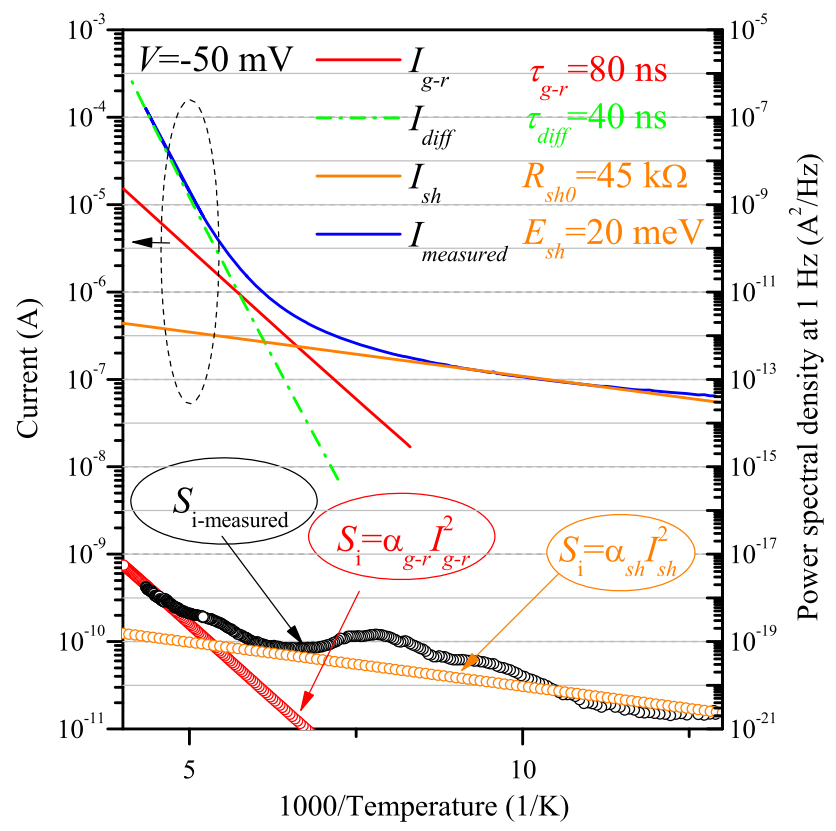

Fig. 6 Power spectral density at $1 \mathrm{~Hz}$ and dark current (decomposed into constituents) versus $1000 / T$ at constant reverse voltage bias $V=50 \mathrm{mV}$

include formulas developed for bulk semiconductor devices. This approach seems to be not fully justified but is sufficient for $1 / \mathrm{f}$ noise modeling due to a good agreement with the experiment. In our dark current modeling procedure, several current components are taken into account, namely: the diffusion $I_{d i f f}$, the bulk g-r $I_{g-r}$, the band-to-band tunneling $I_{b t b}$, the trap-assisted tunneling $I_{t a t}$, and the ohmic shunt $I_{s h}$. The diffusion current and bulk g-r current can be calculated as (Gopal et al. 2008; Sze and Ng 2006): 
Fig. 7 Dark current of the detector and its components versus bias voltage at $T=230 \mathrm{~K}$

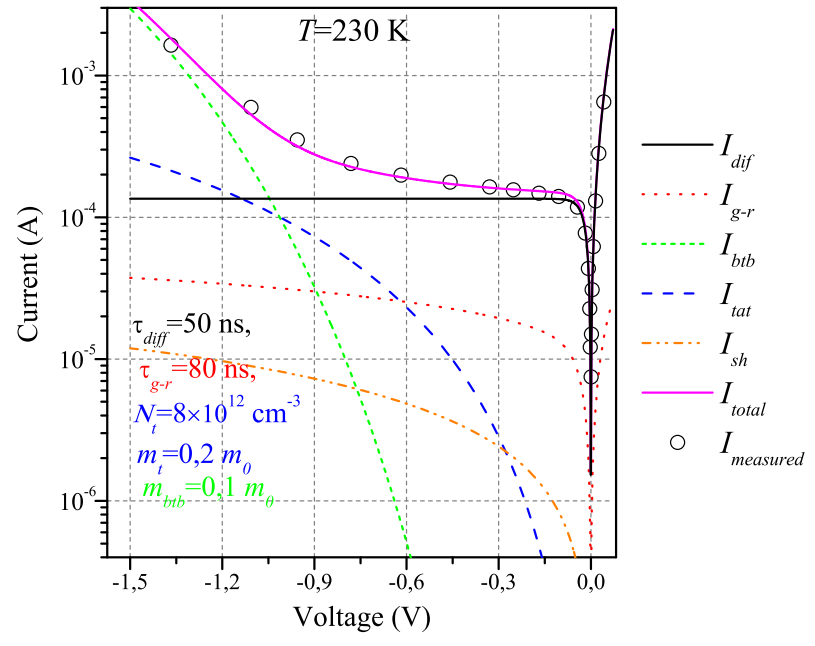

Fig. 8 Predicted and measured 1/f noise p.s.d. at $1 \mathrm{~Hz}$ and their contributions versus total current at $T=230 \mathrm{~K}$. The indexes " $\mathrm{r}$ " and " $\mathrm{f}$ " refer to reverse and forward bias, respectively

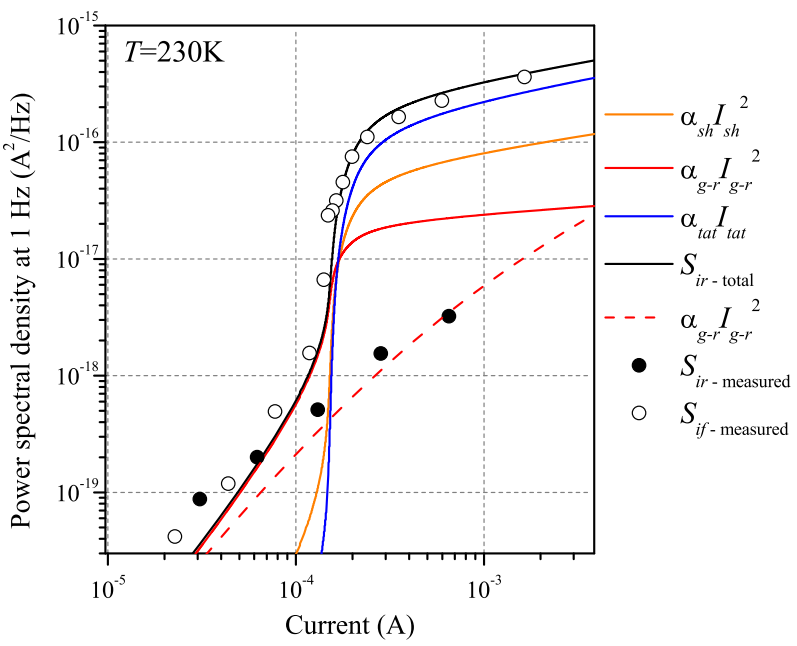

$$
\begin{gathered}
I_{\text {diff }}=\frac{A q n_{i}^{2}}{N_{A}} \sqrt{\frac{k T \mu}{q \tau_{d i f f}}} \tanh \left(\frac{d}{L}\right)\left(\exp \left(\frac{q V}{k T}\right)-1\right), \\
I_{g-r}=\frac{A w q n_{i}}{\tau_{g-r}}\left(\exp \left(\frac{q V}{2 k T}\right)-1\right),
\end{gathered}
$$

where $d$ is the absorber thickness, $\mu, L, \tau_{\text {diff }}$ are the electron mobility, the diffusion length, and the lifetime, respectively; $w$ is the depletion region width, and $\tau_{g-r}$ is the g-r lifetime. Only the lifetimes are the fitting parameters. The depletion region width is calculated with an abrupt junction formula $w=\left(2 \epsilon_{r} \epsilon_{0}\left(V_{b i}-V\right) /\left(q N_{\text {red }}\right)\right)^{1 / 2}$, where the built-in voltage $V_{b i}$ is estimated as $V_{b i}=k T / q \ln \left[N_{D} N_{A} /\left(n_{i}\right)^{2}\right]$, and the intrinsic carrier concentration is calculated as $n_{i}=\left(N_{C} N_{V}\right)^{1 / 2} \exp \left(-E_{g} / 2 k T\right)$. The reduced carrier concentration $N_{\text {red }}=$ 
$N_{A} N_{D} /\left(N_{A}+N_{D}\right) \approx N_{A}$ is roughly $N_{\text {red }}=5 \times 10^{16} \mathrm{~cm}^{-3}$. It is determined from the C-V measurements (see Fig. 3), which were performed at $T=77 \mathrm{~K}$. The $3 \mathrm{D}$ effective densities of states $N_{C}, N_{V}$ were estimated using the value of effective masses: $m_{h h}=0.4 m_{0}$ for heavy holes, and $m_{e}=0.03 m_{0}$ for electrons. The Varshni formula for the temperature dependence of the band gap $E_{g}(T)=234-3.1 \times 10^{-4} T^{2} /(T+270)$ [meV] is implemented with the coefficients reported for $10 \mathrm{ML} / 10 \mathrm{ML}$ InAs/GaSb p-i-n detectors (Klein et al. 2011). The trap-assisted tunneling $I_{t a t}$ and the band-to-band tunneling $I_{b t b}$ can be approximated as (Martyniuk et al. 2012; Yang et al. 2002):

$$
\begin{gathered}
I_{t a t}=\frac{A q^{2} m_{t a t} M^{2} N_{T} V}{8 \pi \hbar^{3}\left(E_{g}-E_{T}\right)} \exp \left(\frac{-4 \sqrt{2 m_{t a t}}\left(E_{g}-E_{T}\right)^{1.5}}{3 q E_{\text {max }} \hbar}\right), \\
I_{b t b}=\frac{A q^{3} E_{\max } V}{4 \pi^{2} \hbar^{2}} \sqrt{\frac{2 m_{b t b}}{E_{g}}} \exp \left(\frac{-4 \sqrt{2 m_{b t b}}\left(E_{g}\right)^{1.5}}{3 q E_{\max } \hbar}\right) .
\end{gathered}
$$

The maximum electric field is calculated with the formula $E_{\text {max }}=\left[2 q N_{\text {red }}\left(V_{b i}-V\right) / \epsilon_{r} \epsilon_{0}\right]^{1 / 2}$. The trap energy $E_{T}$ is assumed as $E_{T}=0.14 \mathrm{eV}$ (Wróbel et al. 2015). The matrix element associated with the trap potential is $M^{2}=10^{-23} \mathrm{eV}^{2} \mathrm{~cm}^{3}$. This value is commonly used for infrared detectors (Rosenfeld and Bahir 1992; Yang et al. 2002). Other symbols in Eqs. (2)-(5) have the usual meaning. The effective tunneling masses $m_{t a t}, m_{b t b}$ and the trap density $N_{T}$ are the fitting parameters. The ohmic-like behavior of $\mathrm{I}-\mathrm{V}$ characteristics can be described by some shunt resistance $R_{s h}$ and the shunt component $I_{s h}$ of the dark current:

$$
I_{s h}=V / R_{s h} .
$$

The shunt resistance can be temperature dependent. This effect can be apparently taken into account with the Arrhenius-like formula:

$$
R_{s h}=R_{s h 0} \exp \left(E_{s h} / k T\right),
$$

where $E_{s h}$ is the activation energy of the shunt current, and $R_{s h 0}$ is the constant.

The material/device parameters used in the dark current modeling are gathered in Table 1.

Table 1 Parameters used for the dark current modeling of the InAs/GaSb-based device (references in brackets)

\begin{tabular}{ll}
\hline$m_{e}$ & $0.03 \cdot m_{0}$ (Imbert et al. 2015; Gopal et al. 2008; Suchalkin et al. 2011) \\
$m_{h}$ & $0.4 \cdot m_{0}$ (Gopal et al. 2008) \\
$\mu_{e}\left[\mathrm{~cm}^{2} / \mathrm{Vs}\right]$ & $11400(T=77 \mathrm{~K})\left(\right.$ Suchalkin et al. 2011), $\mu_{e} \sim T^{-2}$ (Haugan et al. 2005) \\
$\epsilon_{r}$ & $15.4($ Christol et al. 2007) \\
$E_{g}(T)[\mathrm{meV}]$ & $234-3.1 \times 10^{-4} T^{2} /(T+270)$ (Klein et al. 2011) \\
$N_{A}\left[\mathrm{~cm}^{-3}\right]$ & $N_{A} \approx N_{\text {red }}$ (Fig. 3) \\
$d[\mu \mathrm{m}]$ & 2.7 \\
$M^{2}\left[\mathrm{eV}^{2} \mathrm{~cm}^{3}\right]$ & $10^{-23}$ (Yang et al. 2002) \\
\hline
\end{tabular}


Additional aspects of current modeling, i.e., the discussion on electronic transport and fitting parameters in the context of devices physics, were described in our previous paper (Ciura et al. 2016).

\section{1/f Noise modeling}

\subsection{Parameter identification}

The first step of $1 / \mathrm{f}$ noise modeling is the dark current decomposition. Then the fitting procedure should be used to find the 1/f noise coefficients $\alpha$ from the noise p.s.d. versus total current characteristics of the detector. The unambiguous modeling of $1 / \mathrm{f}$ noise or current is possible only if the number of parameters is not too large. This number can be reduced by choosing an appropriate type of the experiment. First of the proposed experiments includes current and 1/f noise measurements/modeling versus voltage bias at a low constant temperature. Then the diffusion and the bulk g-r currents are very low due to a very low intrinsic carrier concentration, so they can be neglected. Second type of the experiments includes measurements/modeling versus temperature at a low constant voltage bias. In that case, both tunneling currents can be neglected due to a small maximum electric field in a junction.

The result of current modeling versus bias voltage at $T=77 \mathrm{~K}$ is presented in Fig. 4. The total current consists of the shunt current, the trap-assisted and the band-to-band tunneling components. The first one dominates in the low bias region, whereas the trapassisted tunneling and the band-to-band tunneling prevail in the medium or the high bias range, respectively. The values of the fitting parameters are shown in Fig. 4. The results of the $1 /$ f noise modeling are presented in Fig. 5, where the p.s.d. at $1 \mathrm{~Hz}$ is plotted versus the total current of the detector. This modeling involves only the fitting of coefficients $\alpha$ to the experimental data, because the dark current components of the detector are taken as in Fig. 4. Note that a very good convergence can be achieved without $1 / \mathrm{f}$ noise associated with the band-to-band tunneling. The 1/f noise p.s.d. follows squared total current $S_{i} \sim I^{2}$ at low current range $\left(I<10^{-6} \mathrm{~A}\right)$. In this range, the $1 / \mathrm{f}$ noise is essentially associated with the shunt current $S_{i}=\alpha_{s h}\left(I_{s h}\right)^{2}$, with the shunt $1 / \mathrm{f}$ noise coefficient $\alpha_{s h}=8 \times 10^{-7}$. For the large current $\left(I>10^{-6} \mathrm{~A}\right)$, the $1 / \mathrm{f}$ noise is associated with the trap-assisted tunneling current $S_{i}=\alpha_{t a t} I_{t a t}$, with the trap-assisted-tunneling $1 / \mathrm{f}$ noise coefficient $\alpha_{\text {tat }}=1.3 \times 10^{-12} \mathrm{~A}$. The curve in Fig. 5 has lower slope for high current due to linear, not squared, relation between current $I_{\text {tat }}$ and 1/f noise. The band-to-band tunneling current does not contribute to the $1 / \mathrm{f}$ noise despite being one order of magnitude larger than current $I_{\text {tat }}$ (see Fig. 4). Consequently, the 1/f noise coefficient of band-to-band tunneling $\alpha_{b t b}$ should be a few orders of magnitude lower than coefficient $\alpha_{\text {tat }}$. For the small currents, the formula $S_{i}=\alpha_{\text {tat }} I_{\text {tat }}$ predicts values of p.s.d. higher than measured. It suggests that, in the ohmic region, the model $S_{i} \sim\left(I_{\text {tat }}\right)^{2}$ is more adequate. This suggestion is confirmed in the theoretical work of Kleinpenning (1983), who has shown that, for the nonlinear detectors, in the ohmic range, the squared dependence between current and 1/f noise is expected.

The prediction of $1 / \mathrm{f}$ noise at an arbitrary bias and temperature requires the knowledge of the remaining coefficient $\alpha_{g-r}$. The estimation of the bulk g-r current $1 / \mathrm{f}$ noise coefficient should be done at higher temperatures, at which this current becomes dominant, and for the low bias where tunneling currents can be neglected. In Fig. 6, the Arrhenius plots for p.s.d. at $1 \mathrm{~Hz}$ (right axis) and the dark current (left axis) can be observed. The 
modeling/measurements were performed at constant reverse voltage bias $V=50 \mathrm{mV}$. The one order of magnitude of the current scale corresponds with the two orders of magnitude of the p.s.d. scale. This makes both the current and the 1/f noise p.s.d. curves parallel if the 1/f noise follows squared current $S_{i} \sim I^{2}$. In Fig. 6, the dark current is resolved into three components: the shunt, the bulk g-r, and the diffusion (see the fitting parameters in Fig. 6). In the low temperature region, the shunt current dominates in the total dark current; however, in the high temperature region, the bulk $\mathrm{g}-\mathrm{r}$ and the diffusion currents prevail. The shunt current is slightly $T$-dependent, whereas the bulk g-r and the diffusion currents are strongly $T$-dependent. In the entire temperature range, the current changes approximately 3.5 orders of magnitude, while $1 / \mathrm{f}$ noise p.s.d. changes approximately four orders of magnitude. It means that squared relation between the total dark current and the $1 / \mathrm{f}$ noise is not fulfilled in the entire range. In Fig. 6 , the shunt current $1 / \mathrm{f}$ noise $S_{i}=\alpha_{s h}\left(I_{s h}\right)^{2}$ is shown. In this formula, there are no adjustable parameters, because $I_{s h}$ is taken as in Fig. 6, and $1 / \mathrm{f}$ coefficient $\alpha_{s h}=8 \times 10^{-7}$ has been previously determined (see Fig. 5). The formula $S_{i}=\alpha_{s h}\left(I_{s h}\right)^{2}$ predicts well the weak $T$-dependence of the $1 /$ f noise in the low temperature region. Small bump in the $1 / \mathrm{f}$ noise can be attributed to the excess $\mathrm{g}-\mathrm{r}$ noise with Lorentzian-like p.s.d. shape. In the high temperature region, there is a significant deviation of the measured p.s.d. from the trend defined by the equation $S_{i}=\alpha_{s h}\left(I_{s h}\right)^{2}$. By the modeling, this deviation can be attributed to the bulk g-r $1 / \mathrm{f}$ noise $S_{i}=\alpha_{g-r}\left(I_{g-r}\right)^{2}$ with fitted $1 / \mathrm{f}$ noise coefficient $\alpha_{g-r}=2 \times 10^{-8}$. No $1 / \mathrm{f}$ noise from the diffusion current is observed. Nevertheless, it does not mean that the diffusion current does not exhibit $1 / \mathrm{f}$ noise, but the small coefficient $\alpha_{\text {diff }}$ makes the $1 / \mathrm{f}$ noise from the diffusion current not observable in these circumstances.

\subsection{1/f Noise model verification}

The calculations of $\mathrm{I}-\mathrm{V}$ characteristic and $1 / \mathrm{f}$ noise p.s.d. were done for $T=230 \mathrm{~K}$ to validate the 1/f noise empirical model. In Fig. 7, the dark current versus bias voltage at this temperature is shown.

Almost all contributions of the dark current are modeled with exactly the same parameters as shown in Figs. 4 and 6, so essentially the bulk model, described in Sect. 3, can predict the value of the dark current at arbitrary voltage bias or temperature with a reasonable accuracy. At $T=230 \mathrm{~K}$, the diffusion current dominates for forward bias and in the low- and mid-voltage range for reverse bias, while tunneling currents become important in the high voltage (reverse) bias. The bulk g-r and especially the shunt current are lower than the diffusion current. Their impact on the dark current characteristics is minor ( $\mathrm{g}-\mathrm{r}$ current) or negligible (shunt current). The key result of the paper is presented in Fig. 8, where the 1/f noise p.s.d. at $1 \mathrm{~Hz}$, calculated with Eq. (1), is plotted versus dark current. In this formula, the dark current contributions are taken as in Fig. 7, whereas 1/f noise coefficients were taken from the independent experiments described above. Therefore, no fitting procedure was used in this step. For the entire forward low-reverse bias, the 1/f noise comes exclusively from the bulk g-r current despite its small contribution to the total current. For the high-reverse bias, the $1 / \mathrm{f}$ noise originates mainly from the trapassisted tunneling and the shunt currents. In the mid-current region $\left(I \approx 1.5 \times 10^{-4} \mathrm{~A}\right)$, all three contributions have the same involvement in the total $1 / \mathrm{f}$ noise. The diffusion current does not contribute to $1 / \mathrm{f}$ noise at any bias. The predicted $1 / \mathrm{f}$ noise characteristic corresponds with the measured one very well. The quite complicated measured characteristic is traced accurately with the $1 / \mathrm{f}$ noise empirical model. 


\section{Conclusions}

Modeling of the dark current for the InAs/GaSb-based superlattice $\mathrm{p}^{+}-\mathrm{p}-\mathrm{n}$ mid-wavelength infrared detector, using relations developed for bulk semiconductor devices, gives the results in good agreement with the experiments. The device $1 / \mathrm{f}$ noise can be accurately modeled with the empirical relation: $S_{1 / f}(f)=\left[\alpha_{s h}\left(I_{s h}\right)^{2}+\alpha_{g-r}\left(I_{g-r}\right)^{2}+\alpha_{t a t} I_{t a t}\right] / f$. The noise coefficient $\alpha$ is the highest for the shunt current $\alpha_{s h}=8 \times 10^{-7}$, while for the bulk $\mathrm{g}_{-}$ $\mathrm{r}$ current the $1 / \mathrm{f}$ noise coefficient is smaller $\left(\alpha_{g-r}=2 \times 10^{-8}\right)$. The $1 / \mathrm{f}$ noise coefficients are temperature-independent. No 1/f noise from the diffusion and the band-to-band tunneling currents is observed. The $1 / \mathrm{f}$ noise contributions from the shunt and the bulk $\mathrm{g}-\mathrm{r}$ currents can be high even if they have very small contribution to the total current of the detector. At high voltage bias, the trap-assisted tunneling contributes to the $1 / \mathrm{f}$ noise; however, there is a linear $S_{i} \sim I_{\text {tat }}$, not squared, relation between the $1 / \mathrm{f}$ noise power spectral density and the trap-assisted-tunneling current.

Acknowledgements This work was partially supported by the National Science Center (Projects Nos. 2014/13/N/ST7/03074 and 2013/11/B/ST7/04341).

Open Access This article is distributed under the terms of the Creative Commons Attribution 4.0 International License (http://creativecommons.org/licenses/by/4.0/), which permits unrestricted use, distribution, and reproduction in any medium, provided you give appropriate credit to the original author(s) and the source, provide a link to the Creative Commons license, and indicate if changes were made.

\section{References}

Bae, S.H., Lee, S.J., Kim, Y.H., Lee, H.C., Kim, C.K.: Analysis of $1 / f$ noise in LWIR HgCdTe photodiodes. J. Electron. Mater. 29(6), 877-882 (2000)

Bajaj, J.: Noise $(1 / f)$ and dark currents in midwavelength infrared PACE-I HgCdTe photodiodes. J. Vac. Sci. Technol. B Microelectron. Nanometer Struct. 10(4), 1617-1625 (1992)

Callewaert, F., Hoang, A.M., Razeghi, M.: Generation-recombination and trap-assisted tunneling in long wavelength infrared minority electron unipolar photodetectors based on InAs/GaSb superlattice. Appl. Phys. Lett. 104(5), 053508 (2014)

Christol, P., Konczewicz, L., Cuminal, Y., Ait-Kaci, H., Rodriguez, J.B., Joullie, A.: Electrical properties of short period InAs/GaSb superlattice. Phys. Status Solidi C 4(4), 1494-1498 (2007)

Ciura, Ł., Kolek, A., Gawron, W., Kowalewski, A., Stanaszek, D.: Measurements of low frequency noise of infrared photodetectors with transimpedance detection system. Metrol. Meas. Syst. 21(3), 461-472 (2014)

Ciura, Ł., Kolek, A., Jureńczyk, J., Czuba, K., Jasik, A., Sankowska, I., Papis-Polakowska, E., Kaniewski, J.: Noise-current correlations in InAs/GaSb type-II superlattice midwavelength infrared detectors. IEEE Trans. Electron Devices 63(12), 4907-4912 (2016)

Czuba, K., Sankowska, I., Jureńczyk, J., Jasik, A., Papis-Polakowska, E., Kaniewski, J.: Influence of Be doping placement in InAs/GaSb superlattice-based absorber on the performance of MWIR photodiodes. Semicond. Sci. Technol. 32(5), 055010 (2017)

Gopal, V., Plis, E., Rodriguez, J.B., Jones, C.E., Faraone, L., Krishna, S.: Modeling of electrical characteristics of midwave type II InAs/GaSb strain layer superlattice diodes. J. Appl. Phys. 104(12), 124506 (2008)

Haugan, H.J., Brown, G.J., Szmulowicz, F., Grazulis, L., Mitchel, W.C., Elhamri, S., Mitchell, W.D.: InAs/ GaSb type-II superlattices for high performance mid-infrared detectors. J. Cryst. Growth 278(1-4), 198-202 (2005)

Imbert, J., Trinite, V., Derelle, S., Jaeck, J., Giard, E., Delmas, M., Carras, M., Haidar, R., Rodriguez, J.B., Christol, P.: Electronic structure of InAs/GaSb superlattice for the modelling of MWIR pin photodiode. Infrared Phys. Technol. 70, 81-86 (2015) 
Khoshakhlagh, A., Myers, S., Kim, H., Plis, E., Gautam, N., Lee, S.J., Noh, S.K., Dawson, L.R., Krishna, S.: Long-wave InAs/GaSb superlattice detectors based on $\mathrm{nBn}$ and pin designs. IEEE J. Quantum Electron. 46(6), 959-964 (2010)

Kinch, M.A., Wan, C.-F., Schaake, H., Chandra, D.: Universal $1 / f$ noise model for reverse biased diodes. Appl. Phys. Lett. 94(19), 193508 (2009)

Klein, B., Plis, E., Kutty, M.N., Gautam, N., Albrecht, A., Myers, S., Krishna, S.: Varshni parameters for InAs/GaSb strained layer superlattice infrared photodetectors. J. Phys. D Appl. Phys. 44(7), 075102 (2011)

Kleinpenning, T.G.M.: On noise and detectivity in reverse-biased pn-junction photodiodes. Phys. B+C 121(1-2), 81-88 (1983)

Martyniuk, P., Wróbel, J., Plis, E., Madejczyk, P., Kowalewski, A., Gawron, W., Krishna, S., Rogalski, A.: Performance modeling of MWIR InAs / GaSb / B-Al ${ }_{0.2} \mathrm{Ga}_{0.8} \mathrm{Sb}$ type-II superlattice nBn detector. Semicond. Sci. Technol. 27(5), 055002 (2012)

Martyniuk, P., Benyahia, D., Kowalewski, A., Kubiszyn, Ł., Stępień, D., Gawron, W., Rogalski, A.: Midwave T2SLs InAs / GaSb single pixel PIN detector with GaAs immersion lens for HOT condition. Solid-State Electron. 119, 1-4 (2016)

Nguyen, T., Musca, C.A., Dell, J.M., Antoszewski, J., Faraone, L.: Dark currents in long wavelength infrared $\mathrm{HgCdTe}$ gated photodiodes. J. Electron. Mater. 33(6), 621-629 (2004)

Peng, R., Jiao, S., Li, H., Zhao, L.: Dark current mechanisms investigation of surface passivation InAs/GaSb photodiodes at low temperatures. J. Alloys Compd. 632, 575-579 (2015)

Rodriguez, J.B., Plis, E., Bishop, G., Sharma, Y.D., Kim, H., Dawson, L.R., Krishna, S.: nBn structure based on InAs/GaSb type-II strained layer superlattices. Appl. Phys. Lett. 91(4), 04351 (2007)

Rosenfeld, D., Bahir, G.: A model for the trap-assisted tunneling mechanism in diffused n-p and implanted $\mathrm{p}^{+}$-p HgCdTe photodiodes. IEEE Trans. Electron Devices 39(7), 1638-1645 (1992)

Suchalkin, S., Belenky, G., Svensson, S.P., Laikhtman, B., Smirnov, D., Tung, L.C., Bandara, S.: In-plane and growth direction electron cyclotron effective mass in short period InAs/GaSb semiconductor superlattices. J. Appl. Phys. 110(4), 043720 (2011)

Sze, S.M., Ng, K.K.: Physics of Semiconductor Devices, 3rd edn. John Wiley and Sons, Hoboken (2006)

van der Ziel, A.: Review of the status of quantum 1/f noise in $\mathrm{p}^{+}-\mathrm{p}$ HgCdTe photodetectors and other devices. J. Vac. Sci. Technol. A 4(4), 2205-2216 (1986)

Wróbel, J., Ciura, Ł., Motyka, M., Szmulowicz, F., Kolek, A., Kowalewski, A., Moszczyński, P., Dyksik, M., Madejczyk, P., Krishna, S., Rogalski, A.: Investigation of a near mid-gap trap energy level in midwavelength infrared InAs/GaSb type-II superlattices. Semicond. Sci. Technol. 30(11), 115004 (2015)

Yang, Q.K., Fuchs, F., Schmitz, J., Pletschen, W.: Investigation of trap-assisted tunneling current in InAs/ (GaIn)Sb superlattice long-wavelength photodiodes. Appl. Phys. Lett. 81(25), 4757-4759 (2002) 